\title{
MORTALIDAD PERINATAL
}

\author{
Sociedad Vallecaucana de obstetricia y Ginecología \\ Jorge Escobar Soto * \\ Arnulfo Gómez Rueda ** \\ Carlos A. Quintero Jaramillo
}

Ha sido un gran acierto de la Sociedad Colombiana de Obstetricia y Ginecología escoger la Mortalidad Peri-Natal como tema oficial de este Congreso, ya que el interés sobre el problema lo ha vuelto objeto principal de discusión, no sólo en los medios médicos y biológicos, sino también en los socio-económicos mundiales.

Este interés se explica fácilmente por la variedad de factores que inciden sobre el crítico período Peri-Natal.

Para las ciencias médico-biológicas es obvia la inquietud en el problema, ya que la rata mundial de peri-mortalidad no muestra el mismo descenso que muestra la mortalidad en otras edades de la vida. A este hecho se añaden
Ios problemas relacionados con la incidencia de prematurez. Aunque las mejores técnicas de cuidados médicos del prematuro han logrado una baja en la rata de mortalidad, también es obvio que falta mucho por estudiar y conocer sobre las causas y prevención del nacimiento prematuro. Este campo importante no ha sido estudiado bajo noımas muy científicas entre nosotros. La complejidad del problema hace su análisis difícil, pues no sólo causas determinantes de "stress' para la madre, como enfermedades astadas o crónicas durante el embarazo, malnutrición, complicaciones propias del embarazo, etc., inciden sobre el nacimiento prematuro, sino gran variedad de factores

* Profesor Asociado, Jefe del Departamento de Obstetricia y Ginecología. Universidad del Valle. Facultad de Medicina.

** Instructor. Departamento de Obstetricia y Ginecología. Universidad del Valle. Facultad de Medicina.

*** Residente. Departamento de Obstetricia y Ginecología. Universidad del Valle. Facultad de Medicina. 
socio-económicos como suficiencia de medios de vida, ilegitimidad, inestabilidad del matrimonio, excesivo número de hijos, etc.

Otro hecho importante que aumenta el interés médico sobre el problema, es la relación estrecha entre afecciones neurológicas y psicológicas y el período peri-natal. Se reconoce ampliamente la frecuencia de lesiones sub-letales de este período de la vida, que inquietan justificadamente a neurólogos y psiquiatras. Este campo de investigación es quizá uno de los más importantes y difíciles con que debemos enfrentarnos. Se espera que un mejor conocimiento de los factores que inciden en la mortalidad peri-natal permitan la prevención y tratamiento de las circunstancias que rodean la aparición de lesiones subletales del período peri-natal.

Dejada así esbozada la importancia del problema, es necesario insistir sobre dos puntos: el primero, la enorme variedad de factores que inciden sobre la mortalidad peri-natal, y segundo, que es más importante el análisis de estos factores más bien que el estudio de un pequeño número de causas bien definidas. Desde luego conocer y analizar estos factores es un problema difícil, ya que sobre el período peri-natal obran hechos que sucedieron con anterioridad, ya sea durante el embarazo o aun antes de la fecundación.

Hace muy difícil el análisis de estos factores tan diversos, la falta de precisión de la observación clínica y patológica. Entre nosotros estas fallas son notorias y hacen la comparación de datos muy equivocada.

\section{Objetivos}

19 Estudiar la mortalidad peri-natal en el Hospital Universitario del Valle, Servicios de Obstetricia y Ginecología para el año comprendido entre el $1^{\mathbf{9}}$ de enero y el 31 de diciembre de 1962, a través de los factores y causas que la determinan.

20 Establecer los factores y las causas de defunción, para conocer los coeficientes respectivos.

3o Investigar la forma de influír sobre los factores y las causas de mortalidad peri-natal en el Servicio de Obstetricia y Ginecología.

\section{Plan para llenar los objetivos}

19 Estudio del problema por medio de la observación y la referencia de trabajos similares.

20 Definiciones y limitaciones para el trabajo: La Organización Mundial de la Salud ha hecho esfuerzos para unificar definiciones y conceptos, así como también ha elaborado cuestionarios para servir de guía en los certificados de defunción. Sin embargo, nosotros no los hemos usado, y no sabemos si se están usando en el país.

Como período peri-natal consideramos un término delimitado arbitrariamente de acuerdo a estadísticas clínicas. El concepto fue inicialmente introducido por Pfaundler en 1936 en una forma un poco confusa; posteriormente fue revaluado, habiéndose dado de él varias definiciones que comprendían variaciones en los límites tanto superior como inferior, y que oscilaban entre 
los 3 a 30 días, aunque la mayoría de los autores usaban el término de 7 días, especialmente después de un concepto dado por Wallgreen's en 1942. El término de 7 días ha sido el más usualmente aceptado, de acuerdo con la decisión tomada por la O. M. S.

El límite inferior ha sido más difícil de establecer. Como norma general no debe haber ningún límite inferior, y todos los productos de la concepción deberían incluírse, aun los embarazos ectópicos, pero como el concepto de mortalidad peri-natal debe tener un sentido práctico, se ha adoptado excluír las primeras 20 semanas de embarazo. Para el período de la 20 a 28 semana, solamente deben tenerse en cuenta los nacidos vivos. Este límite inferior cstará representado en tiempo de la dura. ción de la preñez, expresada en semanas o días a partir del primer día de la última menstruación, o en gramos de peso. En todas las publicaciones de la O. M. S. se ha tomado como límite inferior a la vigésimaoctava semana, y como equivalente a ella los 1.000 gramos de peso.

Para poder llevar a cabo el estudio sobre este tema nos hemos visto forzados a utilizar los conceptos y definiciones que nos han perecido más útiles y más generalmente aceptados. Con este criterio adoptamos las siguiente definición de mortalidad peri-natal: el número de nacidos que mueren antes del parto, durante el parto o en el curso de los primeros 7 días después de! parto, y han pesado al nacer más de 1.000 gramos o entre 501 y 1.000 gramos con suficiente vitalidad para sobrevivir.
Otras definiciones que utilizamos en el presente trabajo son: mortinatos: el producto de la concepción muerto in-útero antes del nacimiento completo.

Muerto neo-natal corresponde a la acaecida desde el nacimiento completo hasta el vigésimoctavo día de vida.

El mortinato lo diferenciamos en muerte-ante-partum, cuando ésta sucede antes de la iniciación del parto, y muerte intra-partum cuando ésta sucede durante el trabajo del parto.

Hemos adoptado también las siguientes definiciones: Aborto, la expulsión del producto de la concepción, de peso inferior a 500 gms. Prematuro, el que pesa 1.001 gramos hasta 2.500 gms. Prematuro previable, el feto que pesa de 501 gms a 1.000 gms, que nace vivo. A término, el que pesa de 2.501 gms en adelante.

Desde el comienzo de nuestro estudio nos dimos cuenta rápidamente que los factores tan variables que a nuestro modo de ver influyen sobre la mortalidad peri-natal se podrán agrupar en dos grandes categorías. La primera, la que envuelve una serie de factores demográficos, y la segunda, la que engloba factores médicos.

\section{FACTORES DEMOGRAFICOS.} Hay que de ja r establecido que estos factores no son causas reales de mue rte peri-natal, sino que influyen sobre ella, ya sea interfiriendo para que el feto no soporte bien el cambio de la vida intrauterina a la extrauterina, o también aumentando la incidencia o empeorando la evolución de enfermedades o condicio- 
nes de la madre. Estos factores demográficos podían dividirse en tres grupos; primero: factores que obran en la madre; segundo: factores que obran en el parto, y tercero: factores que obran en el feto.

Factores que obran en la madre: De estos, son de gran importancia, en este grupo, los factores sociales. Se ha demostrado ampliamente que el nivel económico de la familia, el grado de educación, la clase de vida y de trabajo, así como la dieta que puede proporcionarse, tiene influencia en la incidencia tanto del aborto como del parto prematuro y de la mortalidad peri-natal. Dentro de estos factores sociales también hay que incluír factores éticos, actitud hacia el embarazo, ilegitimidad y otras causas de "stress social".

Otro factor importante de este grupo es la edad de las pacientes. Todos sabemos el aumento de riesgo obstétrico para la mujer de edad avanzada, lo mismo para ella como para el hijo. Los estudios de muchos observadores comprueban este conocimiento.

Así mismo conocemos la paridad de la mujer como un factor de mayor riesgo obstétrico, en el curso del primer embarazo y parto, y en la gran multiparidad. El riesgo de la combinación de edad avanzada y primigravidez es de todos nosotros conocido. Si a estos dos factores agregamos los de "stress", el riesgo será aún peor.

Dentro de este grupo también es necesario mencionar la talla y el peso corporal de la mujer como factores que aumentan la incidencia de mortalidad peri-natal, cuando ellos exceden de los términos más o menos prome- dios. Si además se considera el factor nutricional como causa de anormalidades del peso de las madres, es obvia la importancia de estos factores.

Factores que obran en el parto: Es de importancia conocida la influencia del tamaño del feto sobre la mortalidad peri-natal. Niños demasiado grandes elevan la mortalidad intra-partum. Niños demasiado pequeños influyen con la mortalidad en el post-partum.

Otro importante factor de este grupo es la presentación del feto en el parto. Todos estamos familiarizados con el riesgo obstétrico de las presentaciones de hombro, de la pelvis y de las presentaciones cefálicas anormales o distócicas. Si a estos riesgos se añaden los de tamaño del feto, sabemos también cuántos problemas pueden surgir de las diferentes combinaciones posibles.

Factores que obran en el feto: Entre éstos es necesario señalar el embarazo múltiple como causa del riesgo de mortalidad peri-natal. La frecuencia de bajo peso o de pequeño tamaño de los fetos, la incidencia de complicaciones del embarazo en la madre y de dificultades en el parto hacen que la incidencia de mortalidad peri-natal esté considerablemente aumentada en estos casos.

Otro factor importante y además curioso, ya que no ha tenido una explicación suficiente, es la influencia del sexo. Sabemos que la mortalidad perinatal es mayor en los varones que en las hembras, y diversos autores señalan la proporción de este hecho en cifras que varían de 120/100 hasta $150 / 100$ 


\section{FACTORES DE MORTALIDAD PERINATAL}

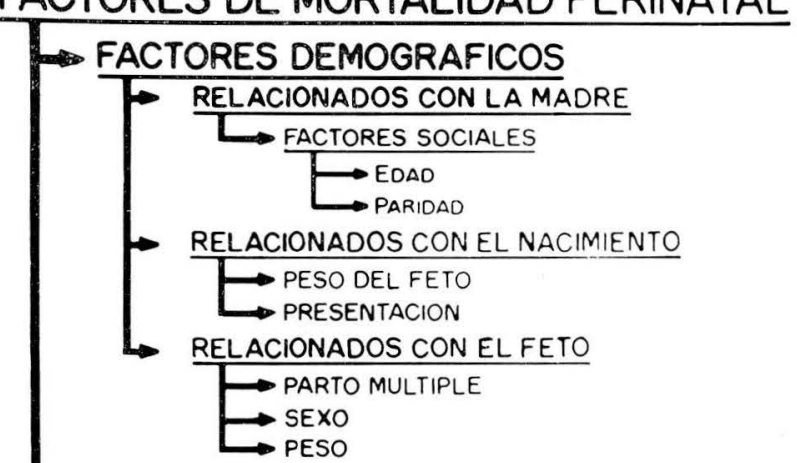

FACTORES MEDICOS - ANOXEMICOS

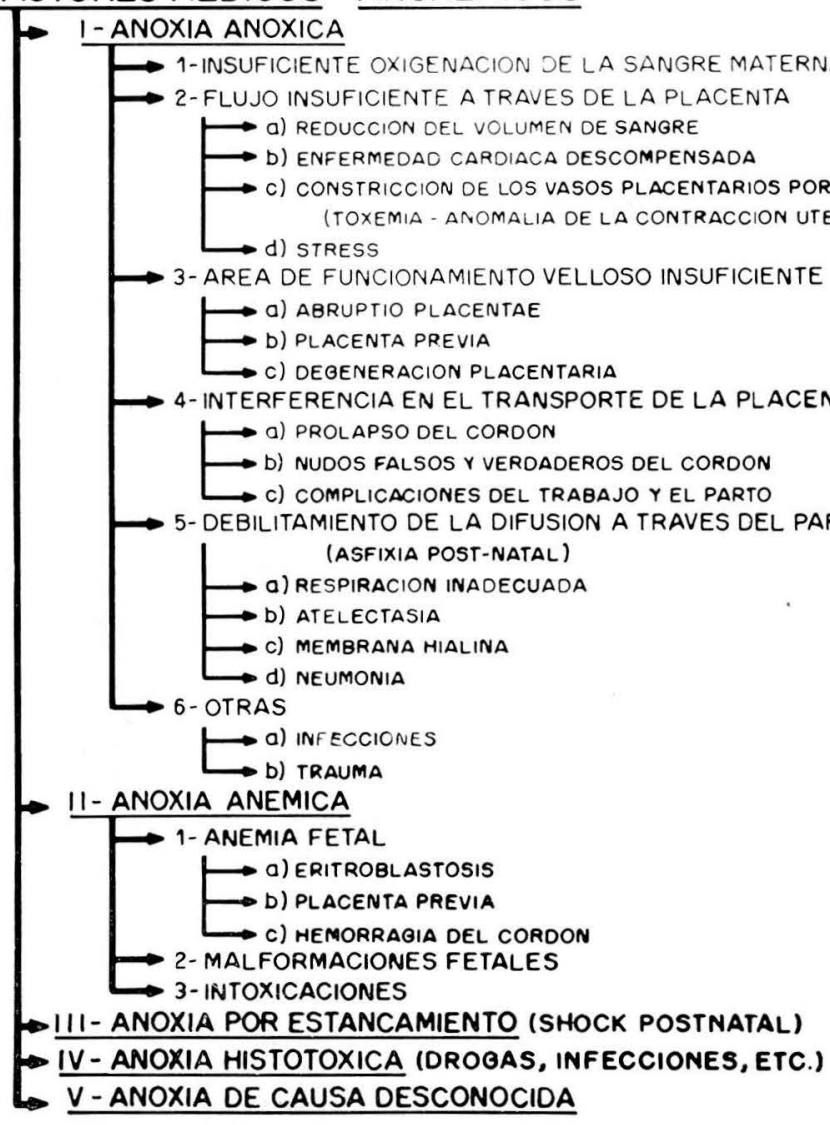

Figura 1 
Peso al nacer: La relación entre el peso del infante al nacer y la mortalidad peri-natal es también bien apreciada por todos nosotros, y las estadísticas mundiales a este respecto son suficientemente concluyentes. La prematurez sigue siendo factor muy importante de mortalidad. Asociada a factores médicos que veremos más adelante, aumenta los riesgos de mortalidad peri-natal en forma por demás conocida para todos nosotros.

Ultimamente ha aparecido un factor importante $y$ nuevo que influencia las cifras de mortalidad peri-natal. Es el infante post-maduro o dismaduro, que en general se asocia a la prolongación del embarazo, y en los cuales se presume la existencia de algún grado de insuficiencia placentaria. Los grados avanzados de esta situación contribuyen a la morti-natalidad, y los grados menos severos, a la mortalidad neo-natal.

\section{FACTORES MEDICOS:}

Vistos así en forma rápida los factores demográficos que influyen sobre el problema, veremos los factores médicos.

Dentro de este grupo hay que englobar muchas enfermedades puramente médicas y síndromes variados, que también por su complejidad hacen difícil el estudio de su influencia sobro la mortalidad peri-natal.

La vida depende de los procesos metabólicos celulares en presencia de oxígeno. Esto sucede tanto en la vida intra-uterina como en el medio extra-uterino. La insuficiencia de oxígeno (hi- poxia) tiene un efecto destructivo sobre la célula misma, y el daño más o menos extenso ocasionado por su aporte insuficiente, lleva a lesiones subletales o letales del organismo, y, por lo tanto, inciden sobre la mortalidad perinatal.

Es de todos conocido el papel de la placenta como órgano de la respiración fetal. Los trastornos en la oxigenación causados por lesiones de la placenta o sus anexos, o por su influencia funcional, son causas de mortalidad fetal bien reconocida por todos. Esta sucede en la gran mayoría de los casos de anoxia, precedida de un período más o menos prolongado de hipoxia. La gran mayoría de los neonatos mueren como resultado de daño causado por hipoxia intrauterina o por hipoxia causada por trastornos de la oxigenación pulmonar. Esta oxigenación pulmonar puede estar interferida por defecto en las estructuras pulmonares, por anomalías de la caja torácica o por daño del centro respiratorio. Sobre este último, la hipoxia intrauterina tiene un efecto destructivo muy especial.

Las fallas en el funcionamiento de la caja toráxica y de las estructuras pulmonares, dependen en mucha parte del grado de madurez del recién nacido. De aquí la gran importancia de la hipoxia en el prematuro.

Se reconocen cuatro grandes grupos de anoxia: Anoxia anóxica, anoxia ané . mica, anoxia por estancamiento y anoxia histotóxica.

Anoxia anóxica: Significa una reducción del abastecimiento de oxígeno para el feto, como resultado de un sumi- 
nistro insuficiente de oxígeno por el organismo materno. Esta situación sucede como resultado de:

19 Oxigenación insuficiente de la sangre materna, como sucede por ejem-plo en las anemias de la madre, intoxicaciones, etc.

$2^{\circ} \quad$ Flujo sanguíneo insuficiente a través de la placenta, como pasa por ejemplo:

a) Reducción del volumen sanguíneo.

b) Enfermedad cardíaca descompensada.

c) Constricción de los vasos placentarios (toxemia, contracciones uterinas anormales, etc.).

d) Reacción de "stress".

$3^{\circ}$ Insuficiencia funcional de las vellosidades coriales, como por ejemplo en:

a) Abruptio placentae, placenta previa.

b) Degeneración placentaria.

$4^{\circ}$ Interferencia con el transporte sanguíneo de la placenta al feto, como en:

a) Procidencia o prolapso de cordón.

b) Nudos falsos o verdaderos o comprensión del cordón umbilical.

c) Complicaciones en el trabajo o en el parto.

Difusión insuficiente del oxígeno a través del parénquima a pulmonar (asfixia neo-natorum), como en: a) Respiración inadecuada.

b) Atelectasia, membrana hialina.

c) Neumonía y bronconeumonía.

Anoxia anémica: Significa un transporte reducido de oxígeno en el feto. Puede ser el resultado de:

19 Anemia fetal como en la eritroblastosis, placenta previa, hemorragia del cordón umbilical, hemorragia por ruptura del seno marginal.

\section{2ํ. Malformaciones.}

\section{$3^{\circ} \quad$ Intoxicaciones.}

Anoxia por estancamiento: Significa reducción de la toma de oxígeno en los tejidos del feto, como sucede en el shock post-natal.

Anoxia histotóxica. Se debe a una oxidación reducida en las céiulas, como puede probablemente suceder bajo la influencia de anestésicos, narcóticos y en algunas infecciones (lúes, septicemia), etc.

Para facilitar el análisis de las causas posibles de mortalidad peri-natal se ha tratado de clasificar estas causas en grupos. Sin embargo, rara vez se logra puntualizar una sola causa como responsable de la muerte. De aquí que en general el análisis clínico y patológico de cada caso debe hacerse en forma tan completa como sea posible y que, además, separadamente uno y otro sean insuficientes para un análisis completamente satisfactorio. Por eso, para aproximarse a la verdad, es necesario el estudio en conjunto de la historia anterior de los padres, revisión cuidadosa de sus antecedentes patológicos y 
personales, evolución del embarazo, del trabajo y del parto, así como el cuadro patológico.

Los diversos modos de agrupar y catalogar las causas de muerte peri-natal han sufrido muy diversos cambios a través de diferentes etapas y autores. Se ha observado una tendencia a agruparlas en la forma siguiente:

1. Malformaciones.

2. Causa obstétrica que incluye distocia, anormalidades de placenta, anexos y lesiones obstétricas.

\section{Otras causas.}

Hubo quienes hicieron de la prematurez un grupo especial, pero este criterio se ha modificado, ya que la prematurez en sí no es una causa de muerte, sino más bien un estado. Algunos, hoy todavía, conservan este grupo para casos de infantes prematuros previables.

\section{3ọ Material y métodos:}

a) Marco de trabajo: Se hizo en el Departamento de Obstetricia y Ginecología una revisión del número de historias obstétricas, en el tiempo comprendido entre el $1^{\circ}$ de enero y el 31 de diciembre de 1962.

b) Sistema de recolección del dato: Se tomó una muestra del $10 \%$ del total de historias por muestreo sistemático.

c) Datos seleccionados: Fueron los siguientes: Número de historia, edad, estado civil, gravidez y paridad, control prenatal, enfermedades intercurrentes, concomitantes y patología asocia- da en la madre; complicaciones del trabajo y del parto características del mismo, intervenciones; peso del feto, sexo, estado al nacer, estado al llegar al hospital, tiempo de vida; patología del neo-nato, hallazgos de autopsia, si la hubo; malformaciones y post-madurez.

Se clasificaron las historias en nacimientos prematuros y a término, y éstos a su vez en neo-natos hasta el 70 día y mortinatos.

d) Pruebas de significancia estadística: Se aplicaron las pruebas del $\mathrm{CHi}$ cuadrado, el error normal de la diferencia de los promedios y su relación de la variación al universo.

Los datos obtenidos en nuestro análisis representan únicamente lo que sucede en nuestra institución, y no puede generalizarse ni para la ciudad de $\mathrm{Ca}-$ li, ni para el Departamento del Valle, ni mucho menos para todo el país. Nuestra institución es el único hospital de caridad de la ciudad con servicio obstétrico y recoge casos no sólo de la ciudad sino de sus alrededores. A causa de la excesiva demanda de hospitalización, muchos casos normales no buscan nuestros servicios, y en cambio de los casos obstétricos complicados la mayoría nos lo solicitan. De aquí que como base debe considerarse que hay una selección de casos, que lleva a error toda comparación con entidades similares.

\section{Nuestras series:}

El cuadro No 1 muestra el marco de trabajo sobre el cual se presenta este estudio. 
CUADRO N: 1

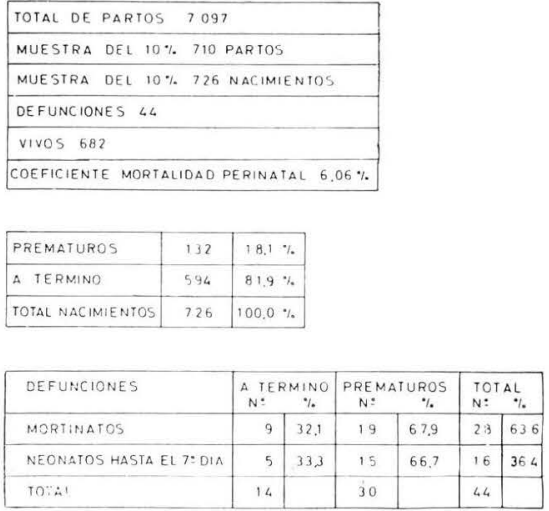

En él aparece un total de 7.097 partos, de los cuales se tomó el $10 \%$ como muestra, que corresponde a 710 partos. Estos dieron lugar a 726 nacimientos. En este grupo encontramos 44 defunciones y 682 vivos. Estas cifras nos dan un coeficiente de mortalidad peri-natal de $6.06 \%$.

En la muestra de 726 nacimientos, encontramos 132 nacidos prematuramente, correspondientes al $18,1 \%$ y 594 a término, correspondientes al $81.9 \%$.

De las 44 defunciones, 28 corresponden a mortinatos y 16 a neonatos hasta el séptimo día, lo que significa un $63.6 \%$ para los primeros y un $36.4 \%$ para los segundos.

De los 28 mortinatos, 32.1\% casos) corresponden a nacidos a término, y $67.9 \%$ (19 casos), a prematuros. De 16 néonatos, hasta el $7^{\circ}$ día, $33.3 \%$ ( 5 casos) corresponden a néonatos a término, y $66.7 \%$ (15 casos), a prematuros néonatos.

\section{FACTORES DEMOGRAFICOS:}

Estado civil: En el cuadro No 2 se presenta en la parte superior un análisis de la distribución de los 726 nacimientos con relación al estado civil de la madre. Se observa que el $48.2 \%$ de nuestros casos pertenecen a matrimonios bien establecidos, mientras el $51.8 \%$ corresponden a pacientes cuya situación matrimonial podría suponer un factor de "stress' social.

Al analizar la mortinatalidad, se anota que de las 44 defunciones, $52 \%$ (23 casos) corresponden a casadas y $48 \%$ a no casadas (21 casos). Estas cifras, desde el punto de vista estadístico, nos demuestran que en nuestro medio la ilegitimidad no tiene influencia en la mortalidad peri-natal.

\section{Edad: Cuadro Nọ 3.}

La edad de la madre ha sido considerada factor importante que influye sobre el problema. En el cuadro No 3, parte superior, se analiza la distribución de nuestros 726 casos, de acuerdo a grupos convencionales de edad, que comprenden madres desde menos de 15 años hasta 40 o más años.

Nos interesa saber si la mujer de edad avanzada significa un mayor riesgo de mortalidad peri-natal. En la parte inferior observamos que sobre 650 hijos de madres de menos de 35 años, murieron 36 , correspondientes al $5.5 \%$, y de 76 casos de hijos de madres de más de 35 años, 8 murieron, correspondientes al $10.5 \%$. Es de anotar que en el $89.5 \%$ de nuestros nacimientos, la madre era menor de 35 años. 


\begin{tabular}{|c|c|c|c|c|c|c|c|c|c|c|c|}
\hline \multirow{3}{*}{\multicolumn{2}{|c|}{ ESTADO CIVIL }} & \multicolumn{6}{|c|}{ DEFUNCIONES } & \multicolumn{3}{|c|}{ VIVOS } & \multirow{3}{*}{ TOTAL } \\
\hline & & \multicolumn{3}{|c|}{ PREMATUROS } & \multicolumn{3}{|c|}{ A TERMINO } & \multirow{2}{*}{ 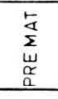 } & \multirow{2}{*}{$\begin{array}{l}\Sigma \\
\alpha \\
w \\
\leftarrow \\
\leftarrow \\
\end{array}$} & \multirow{2}{*}{\begin{tabular}{|l} 
इ \\
\\
$\circ$ \\
$\circ$ \\
on \\
\end{tabular}} & \\
\hline & & MORTINAT & NEONAT & SUB TOTAL & MORTINAT & NEONAT & SUB TOTAL & & & & \\
\hline SOLTERA & 1 & 3 & 1 & 4 & 3 & & 3 & 21 & 152 & 173 & 180 \\
\hline CASADAS & 2 & 12 & 4 & 16 & 3 & 4 & 7 & 45 & 282 & 327 & 350 \\
\hline VIUDA & 3 & & & & & & & & 8 & 8 & 8 \\
\hline UNION LIBRE & 4 & 4 & 6 & 10 & 3 & 1 & 4 & 36 & 138 & 174 & 188 \\
\hline TOTAL & & 19 & 11 & 30 & 9 & 5 & 14 & 102 & 580 & 682 & 726 \\
\hline
\end{tabular}

\begin{tabular}{|c|c|c|c|c|c|c|}
\hline \multirow{3}{*}{$\frac{\text { ESTADO CIVIL }}{\text { CASADAS }}$} & \multicolumn{4}{|c|}{ DEFUNCIONES } & \multirow{2}{*}{\multicolumn{2}{|c|}{ TOTAL }} \\
\hline & \multicolumn{2}{|c|}{ PREMAT } & \multicolumn{2}{|c|}{ A TERMINO } & & \\
\hline & 16 & $533 \%$ & 7 & $50 \%$ & 23 & \\
\hline NO CASADAS & 14 & $467 \%$ & 7 & $5 ? \cdot$ & 21 & 477 \\
\hline TOTAL & \multicolumn{2}{|c|}{30} & \multicolumn{2}{|c|}{14} & \multicolumn{2}{|c|}{44} \\
\hline
\end{tabular}

\section{Paridad:}

Seleccionamos nuestros casos en 3 grupos (cuadro No 4), de acuerdo con el número de partos a que correspondía el embarazo que estudiamos. Estos grupos fueron: $1^{\mathrm{O}}$ Pacientes de paridad 0 a 2; 2o Paridad 3 a 5, y 3ํ Paridad de más de 6.

En el primer grupo encontramos 76 prematuros, de los cuales murieron el $17.1 \%$ (13 casos); 302 a término, de los cuales murieron el $2 \%$ ( 6 casos).

En el segundo grupo: 32 casos de prematuros, con mortalidad del $28.1 \%$ (9 casos) y 189 a término, con mortalidad del $0.5 \%$ (1 caso)..
En el tercer grupo: 24 prematuros con mortalidad del $33.3 \%$ (8 casos) y 103 a término, con mortalidad del $6.8 \%$ ( 7 casos ).

Hechas las pruebas de significancia encontramos que para los prematuros el riesgo de mortalidad era mayor para los del grupo de grandes multipa.ras que para los del grupo primero, no encontrando significancia en el riesgo de mortalidad entre el grupo primero y el segundo, ni entre éste y al tercero. En los a término, encontramos, que el mayor riesgo de mortalidad está en el grupo de las grandes multíparas y disminuye para los del grupo de paridad 0 a 2 en relación con los del grupo intermedio, que es donde existe el menor riesgo. 
C U A D RO Nㅇ 3

\begin{tabular}{|c|c|c|c|c|c|c|c|c|c|c|c|c|}
\hline & \multirow{3}{*}{$E D A D$} & & \multicolumn{6}{|c|}{ DEFUNCIONES } & \multicolumn{3}{|c|}{ VIVOS } & \multirow{3}{*}{ TOTAL } \\
\hline & & & \multicolumn{3}{|c|}{ PREMATUROS } & \multicolumn{3}{|c|}{ A TERMINO } & \multirow{2}{*}{ 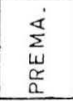 } & \multirow{2}{*}{$\begin{array}{l}\sum \\
\alpha \\
w \\
\leftarrow \\
4\end{array}$} & \multirow{2}{*}{$\begin{array}{l}5 \\
\circ \\
\stackrel{5}{0} \\
\stackrel{0}{5} \\
\end{array}$} & \\
\hline & & & MORTIN. & NEON & $S \cup B-T$ & MORTIN & NEON & $S \cup B T$ & & & & \\
\hline-15 & $A \overline{N O S}$ & 1 & & & & & & & & 1 & 1 & 1 \\
\hline 15 & -19 & 2 & 3 & 2 & 5 & 3 & 1 & 4 & 21 & 104 & 125 & 134 \\
\hline 20 & -24 & 3 & 4 & 1 & 5 & 1 & 1 & 2 & 35 & 211 & 246 & 253 \\
\hline $25-$ & -20 & 4 & 3 & 4 & 7 & 1 & & 1 & 24 & 130 & 154 & 162 \\
\hline $30-$ & -34 & 5 & 6 & 3 & 9 & ? & 2 & 3 & 14 & 74 & 88 & 100 \\
\hline $35-$ & -39 & 6 & 1 & 1 & 2 & 1 & & 1 & 3 & 52 & 55 & 58 \\
\hline+40 & $A \bar{N} O S$ & 7 & 2 & & 2 & 2 & 1 & 3 & 5 & 8 & 13 & 18 \\
\hline \multicolumn{3}{|c|}{ TOTAL } & 19 & 11 & 30 & 9 & 5 & 14 & 102 & 580 & 682 & 726 \\
\hline
\end{tabular}

\begin{tabular}{|c|c|c|c|}
\hline \multirow{2}{*}{$\begin{array}{l}\text { GRUPOS } \\
\text { DE } \\
\text { HISTORIA }\end{array}$} & \multicolumn{2}{|c|}{ EDADES } & \multirow[b]{2}{*}{ TOTAL } \\
\hline & -35 & 435 & \\
\hline VIVOS & \begin{tabular}{l|l|l|}
$6145 \%$ \\
\end{tabular} & \begin{tabular}{l|l|}
68 & $695 \%$ \\
\end{tabular} & 682 \\
\hline MUERTOS & \begin{tabular}{l|l}
36 & $55 \%$ \\
\end{tabular} & $8 \quad 105 \%$ & \\
\hline TOTAL & \begin{tabular}{|l|l|}
$6505 \%$ \\
\end{tabular} & $76105 \%$ & 726 \\
\hline
\end{tabular}

CUAURUN: 4

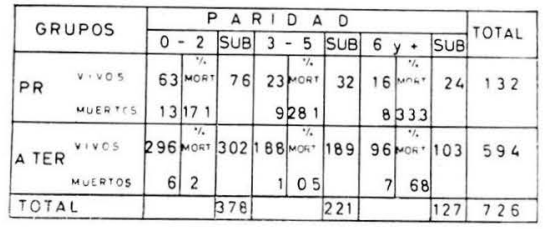

CUADRO N=5

\begin{tabular}{|c|c|c|c|c|c|c|c|c|}
\hline \multirow{2}{*}{ GRUPOS } & \multicolumn{7}{|c|}{ GRA V I DEZ } & \multirow[b]{2}{*}{ TOTAL } \\
\hline & $1-2$ & SuB & 3 & -5 & SuB & $6 y \cdot$ & SUB & \\
\hline 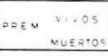 & \begin{tabular}{r|r|}
59 & 132 \\
9 & 132 \\
\end{tabular} & 68 & $\begin{array}{l}21 \\
12 \\
\end{array}$ & 364 & 33 & \begin{tabular}{|l|l|l|}
2 & 2 & 0.0 \\
9 & 2903 \\
\end{tabular} & 31 & 132 \\
\hline A tep Wues & \begin{tabular}{|r|r|}
273 & 4 \\
5 & 18 \\
\end{tabular} & 278 & $\begin{array}{r}186 \\
2 \\
\end{array}$ & 106 & 188 & \begin{tabular}{|r|r|}
221 & \\
7 & 55 \\
\end{tabular} & 128 & 594 \\
\hline TOTAL & & 346 & & & 221 & & 159 & 725 \\
\hline
\end{tabular}

Gravidez: (Cuadro No 5).

Hicimos una distribución similar a la paridad en tres grupos, y encontramos los siguientes resultados:

En el primer grupo: 68 prematuros, con una mortalidad de $13.2 \%$ (9 casos), y 278 a término con una mortalidad del $1.8 \%$ (5 casos).

En el segundo grupo: 33 prematuros, con una mortalidad del $36.4 \%$ (12 casos), y 188 a término, con una mortalidad del $1.06 \%$ (2 casos).

En el tercer grupo: 31 prematuros, con mortalidad del $29.03 \%$ ( 9 casos), 
y 128 a término con mortalidad del $5.5 \%$ (7 casos).

Hechas las pruebas de significancia, encontramos que para los prematuros existe el mayor riesgo de mortalidad en el grupo intermedio, relacionado con el primer grupo y también mayor riesgo de mortalidad entre el $3^{\circ}$ y el $1^{\circ}$, no encontrando diferencia en el riesgo existente entre el $3^{\circ}$ y el intermedio. Para los a término encontramos que el mayor riesgo estaba en los del tercer grupo, en relación con los otros dos grupos, no encontrando diferencia significante en el riesgo de mortalidad entre el grupo primero y el segundo.

Control prenatal: (Cuadro № 6).

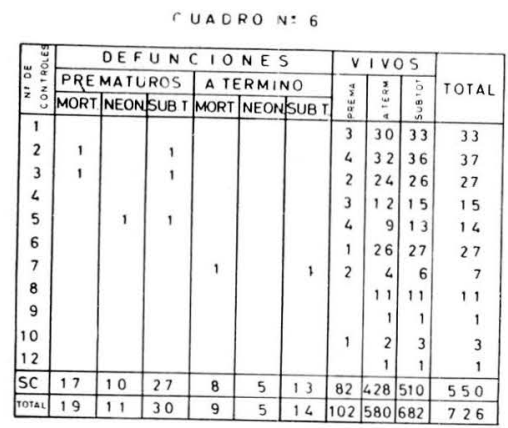

\begin{tabular}{|c|c|c|c|c|c|c|c|c|}
\hline \multirow{3}{*}{\begin{tabular}{|c|} 
CONTROL \\
PRENATAL \\
SIN \\
\end{tabular}} & \multicolumn{4}{|c|}{ DEFUNCIONES } & \multicolumn{3}{|c|}{ VIVOS } & \multirow[b]{2}{*}{ TOTAL } \\
\hline & \multicolumn{2}{|c|}{ PREM } & \multicolumn{2}{|c|}{ A TER } & \multicolumn{2}{|c|}{ PREM } & ATER & \\
\hline & 27 & 90 & 13 & 93 & 82 & 796 & \begin{tabular}{ll|l|l|l|l|}
428 & 738
\end{tabular} & 55075.8 \\
\hline $\mathrm{CON}$ & 3 & 10 & 1 & 7 & 20 & 204 & 15226.2 & $176 \quad 242$ \\
\hline TOTAL & 30 & & 14 & & 102 & & 580 & 726 \\
\hline
\end{tabular}

B

\begin{tabular}{|c|c|c|c|c|c|c|c|}
\hline \multirow[b]{2}{*}{ CONTROL } & \multicolumn{4}{|c|}{ DEFUNCIONES } & \multicolumn{2}{|c|}{ VIVOS } & \multirow[b]{2}{*}{ TOTAL } \\
\hline & $P R$ & EM & $A T$ & ERM & PRE & ATER & \\
\hline SIN & $2^{n+7} 7$ & 49 & 13 & $2 \%$ & 82 & 428 & 550 \\
\hline CON & 3 & 17 & 1 & 06 & 20 & 152 & 176 \\
\hline TOTAL & 30 & & 14 & & 102 & 580 & 726 \\
\hline
\end{tabular}

Se ha creído siempre que un control prenatal adecuado disminuye las cifras de casos obstétricos complicados $y$, por lo tanto, la mortalidad peri-natal.

En el cuadro № 6 observamos cómo de 726 nacimientos, 550, que representan el $76 \%$, no buscaron control prenatal. El resto, 176 que representan el $24 \%$, buscaron control prenatal.

$\mathrm{Al}$ analizar la mortalidad, relacionada con el control, observamos que de 30 prematuros muertos, el 90\% (27 casos) no buscaron control prenatal.

De 14 defunciones a término, el 93\% (13 casos) no buscaron control prenatal.

Analizada la mortalidad peri-natal, con relación al número de casos (550) que no tuvieron control prenatal, observamos que el $4.9 \%$ (27 casos) corresponde a defunciones de prematuros, y que $2.4 \%$ (13 casos) corresponden a defunciones a término.

Comparando estas cifras con las obtenidas de pacientes que sí tuvieron control prenatal, observamos que $1.7 \%$ ( 3 casos) corresponden a prematuros, y $0.6 \%$ corresponden a pacientes a término.

Sexo: (Cuadro No 7).

CUADRO N:?

\begin{tabular}{|c|c|c|c|c|c|c|c|c|c|c|}
\hline \multirow{3}{*}{$S E \times O$} & \multicolumn{6}{|c|}{ DEFUNCIONES } & \multicolumn{3}{|c|}{ VIVOS } & \multirow{3}{*}{ TOTAL } \\
\hline & \multicolumn{3}{|c|}{ PREMATUROS } & \multicolumn{3}{|c|}{$\triangle$ ATERMINO } & & \multirow{2}{*}{\begin{tabular}{|l|l}
5 \\
2 \\
2 \\
2
\end{tabular}} & \multirow[b]{2}{*}{ 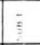 } & \\
\hline & MORT & NEON & $5 \cup 8$ & MORT & NEO & SUBT & & & & \\
\hline & 11 & 5 & 16 & 6 & 2 & 8 & 46 & 305 & 357 & $17 \%$ \\
\hline & 8 & 6 & 14. & 3 & & 6 & 56 & 275 & 331 & 3514 \\
\hline TOTA & 19 & 11 & 30 & 9 & ( & 16 & 102 & 580 & 682 & 726 \\
\hline
\end{tabular}

\begin{tabular}{|l|l|l|l|l|l|l|}
\hline SEXO & MUERTOS & VI VOS & TO TAL \\
\hline HOMBRES & 24 & 54 & 5 & 351 & 515 & 375 \\
MUJERES & 20 & 45 & 5 & 331 & 485 & 351 \\
\hline TOTAL & 44 & & 682 & & 726 \\
\hline
\end{tabular}


En nuestro estudio, aparecen como resultado del nacimiento $51.7 \%$ de productos de sexo masculino (375 casos), y $48.3 \%$ (351 casos) de sexo femenino, como se observa en la parte superior del cuadro 7.

En la parte inferior se observa que de 44 muertos, el $54.5 \%$ corresponde a productos de sexo masculino (24 casos) y $45.5 \%$ a sexo femenino ( 20 casos ).

Estas cifras nos dan una proporción de 120 hombres contra 100 mujeres.

\section{Peso: (Cuadro Nọ 8).}

La parte superior del cuadro No 8, muestra nuestros hallazgos en relación con el peso fetal al nacimiento.

Analizando este cuadro, encontia.mos, que de 30 prematuros muerco: $83.3 \%$ (25 casos) corresponden a pesos entre 501 y 2.000 gramos, como se ve en el cuadro $8 \mathrm{~A}$. Así mismo, $16.7 \%$ ( 5 casos) corresponden a prematuros muertos entre 2.001 y 2.500 gramos. De los 14 muertos a término, $71.4 \%$ ( 10 casos) corresponden a fe. tos entre 2.501 y 3.500 gramos, $28.6 \%$ (4 casos) corresponden a fetos de más de 3.501 gramos.

En el cuadro $8 \mathrm{~B}$, correspondiente al grupo de prematuros, se observa que existe una clara disminución en la mor. talidad, relacionada con el aumento de peso, y observamos que el mayor porcentaje corresponde al grupo entre 501 y 1.000 gramos $(83.3 \%)$, y disminuye hasta un $6.8 \%$ para los de 2 (ji) a 2.500 gramos.

En el cuadro $8 \mathrm{C}$, que corresponde a los prematuros neo-natos, en que el peso al nacer tiene una gran significancia para su sobrevivencia, vemos cómo hay una manifiesta disminución en la mortalidad, relacionando los de menor peso $80 \%$ (501 a 1.0000 gramos) y los de mayor peso 6.8\% (2.00: a 2.500 gramos).

En el cuadro No $8 \mathrm{D}$, que corre:ponde a los casos a término, vemos que para los pesos de 2.501 a 3.500 gramos la mortalidad es de un $0.2 \%$ ( 10 casos), y que en los de 3.501 y más, la mortalidad es del $3.5 \%$ (4 casos).

\section{Presentación: (Cuadro No 9).}

El cuadro No 9 nos muestra la incidencia de las diferentes presentaciones, de acuerdo a la distribución en prematuros y a término, vivos y muertos.

En el cuadro No 9 B, observamos la incidencia de mortalidad de acuerdo a las diferentes presentaciones. Vemos cómo en las presentaciones de vértice (676) casos encontramos un $4 \%$ de mortalidad (27 casos); para las presentaciones de cara ( 3 casos), y un $33 \%$ ( 1 caso); en pelvis, $34.5 \%$ (10 casos), y en hombro un $35.3 \% \quad$ (6 casos).

Vemos que el riesgo de mortalidad entre las presentaciones de cara, pelvis $y$ hombro es sensiblemente igual, y es mayor en comparación con las presentaciones de vértice.

\section{Tipo de parto: (Cuadro No 10).}

En el cuadro No 10 observamos los diferentes tipos de parto, de acuerdo a cada uno de los grupos analizados. 
Cuadro Nọ 8

\begin{tabular}{|c|c|c|c|c|c|c|c|c|c|c|}
\hline \multirow{3}{*}{$\begin{array}{l}\text { PESO } \\
\text { Grs }\end{array}$} & \multicolumn{6}{|c|}{ DEFUNCIONES } & \multicolumn{3}{|c|}{$\mathrm{VIVOS}$} & \multirow{3}{*}{ TOTAL } \\
\hline & \multicolumn{3}{|c|}{ PREMATUROS } & \multicolumn{3}{|c|}{ A TERMINO } & \multirow{2}{*}{$\begin{array}{l}\sum \\
\omega \\
\alpha \\
a \\
a\end{array}$} & \multirow{2}{*}{$\begin{array}{l}\alpha \\
w \\
r \\
a\end{array}$} & \multirow{2}{*}{$\begin{array}{l}5 \\
\infty \\
0 \\
0\end{array}$} & \\
\hline & MORTI & NEON & $S \cup B T$ & MORT & NEON & $S \cup B T$ & & & & \\
\hline 501.1000 & 1 & 4 & 5 & & & & 1 & & 1 & 6 \\
\hline 1.001 .1500 & 5 & 5 & 10 & & & & 11 & & 11 & 21 \\
\hline $1501-2000$ & 8 & 2 & 10 & & & & 21 & & 21 & 31 \\
\hline $2001-2500$ & 5 & & 5 & & & & 69 & & 69 & 74 \\
\hline 25013.000 & & & & 3 & 2 & 5 & & 197 & 197 & 202 \\
\hline $3001-3.500$ & & & & 3 & 2 & 5 & 0 & 272 & 272 & 277 \\
\hline $3.501-4000$ & & & & 2 & & 2 & & 92 & 92 & 94 \\
\hline $4001-4500$ & & & & & & & & 17 & 17 & 17 \\
\hline $4.50 i-5000$ & & & & 9 & & 1 & & 2 & 2 & 3 \\
\hline 5.000 y mas & & & & & 1 & 1 & & & & 1 \\
\hline TOTAL & 19 & 11 & 30 & 9 & 5 & 14 & 102 & 580 & 682 & 726 \\
\hline
\end{tabular}

A

\begin{tabular}{|r|c|r|r|r|r|r|r|}
\hline PESO Grs. & PREMATUROS & \multicolumn{2}{|c|}{ A TERMINO } & \multirow{2}{*}{ TOTAL } \\
\cline { 2 - 6 } & VIVOS & MUERTOS & VIVOS MUERTOS & \\
\hline $501-2000$ & 33 & 25 & $83 \%$ & & & $\%$ & 58 \\
$2001-2500$ & 69 & 5 & 16.7 & & & & 74 \\
$2501-3500$ & & & & 469 & 10 & 71.4 & 479 \\
3501 y mas & & & & 111 & 4 & 28,6 & 115 \\
\hline TOTAL & 102 & 30 & 580 & 14 & 726 \\
\hline
\end{tabular}

\begin{tabular}{|c|c|c|c|c|c|c|c|c|}
\hline & \multirow{2}{*}{\multicolumn{7}{|c|}{ GRUPOS DE PESO }} & \multirow[b]{3}{*}{ TOTAL } \\
\hline \multirow{2}{*}{$\frac{B}{\text { PREMAT }}$} & & & & & & & & \\
\hline & \multicolumn{2}{|c|}{\begin{tabular}{|l|}
$501 \quad \mathrm{a}$ \\
1.000 \\
\end{tabular}} & $\begin{array}{l}1001 a^{2} \\
1500 \\
\end{array}$ & \multicolumn{2}{|c|}{\begin{tabular}{l|l|}
$150 \mathrm{a}^{\mathrm{a}}$ \\
$20000^{2}$ \\
\end{tabular}} & \multicolumn{2}{|c|}{\begin{tabular}{|l|}
$2001 \mathrm{a}$ \\
2500 \\
\end{tabular}} & \\
\hline MUERTOS & 5 & $83 \% 3$ & $100 \% 6$ & 10 & 32,3 & 5 & 6.8 & 30 \\
\hline VIVOS & 1 & 16.7 & 1152.4 & 21 & 67,8 & 69 & 93,2 & 102 \\
\hline TOTAL & 6 & & 21 & 31 & & 74 & & 132 \\
\hline
\end{tabular}

\begin{tabular}{|c|c|c|c|c|c|c|c|c|c|}
\hline \multirow{3}{*}{$\begin{array}{l}\text { PREMNEON. } 7^{\circ} \text { dia } \\
\text { MUERTOS } \\
\text { VIVOS }\end{array}$} & \multicolumn{8}{|c|}{ GRUPOS DE PESO } & \multirow[b]{2}{*}{ TOTAL } \\
\hline & \multicolumn{2}{|c|}{$\begin{array}{r}501 \mathrm{a} \\
1000 \\
\end{array}$} & \multicolumn{2}{|c|}{\begin{tabular}{|l|}
1001 \\
1.500
\end{tabular}} & \multicolumn{2}{|c|}{\begin{tabular}{|l|}
$1501 \mathrm{a}$ \\
$2000^{\circ}$ \\
\end{tabular}} & \multicolumn{2}{|c|}{\begin{tabular}{|l|l|}
$2001 a^{2}$ \\
2500 \\
\end{tabular}} & \\
\hline & $\begin{array}{l}4 \\
1\end{array}$ & $\begin{array}{l}80 \% \\
20 \%\end{array}$ & $\begin{array}{r}5 \\
11\end{array}$ & $\begin{array}{l}31 \% 3 \\
68.7\end{array}$ & $\begin{array}{r}2 \\
21\end{array}$ & $\begin{array}{r}\% \\
8.7 \\
91.3\end{array}$ & $\begin{array}{r}5 \\
69\end{array}$ & $\begin{array}{r}\% .8 \\
93.2\end{array}$ & $\begin{array}{r}16 \\
102\end{array}$ \\
\hline TOTAL & 5 & & 16 & & 23 & & 74 & & 118 \\
\hline
\end{tabular}

D

\begin{tabular}{|c|c|c|c|c|c|}
\hline & GRL & IPOS & $D E$ & PESO & \\
\hline ATERMINO & \begin{tabular}{|ll}
2 & 5 \\
3 & 5 \\
\end{tabular} & 60 & 350 & Oymas & TOTAL \\
\hline MUERTOS & 10 & 0.2 & 4 & 35 & 14 \\
\hline VIVOS & 469 & 99.8 & 111 & 965 & 580 \\
\hline TOTAL & 479 & & 115 & & 594 \\
\hline
\end{tabular}


CUADRO Nㅇ

\begin{tabular}{|c|c|c|c|c|c|c|c|c|c|c|}
\hline \multirow{3}{*}{ P.RESENTACION } & \multicolumn{6}{|c|}{ DEFUNCIONES } & \multicolumn{3}{|c|}{ VIVOS } & \multirow{3}{*}{ TOTAL } \\
\hline & \multicolumn{3}{|c|}{ PREMATUROS } & \multicolumn{3}{|c|}{ ATERMINO } & \multirow{2}{*}{$\begin{array}{l}\sum \\
w \\
\alpha \\
\alpha\end{array}$} & \multirow{2}{*}{$\begin{array}{l}c \\
\stackrel{c}{w} \\
\leftarrow \\
<\end{array}$} & \multirow{2}{*}{$\begin{array}{l}r \\
\infty \\
\vec{\omega} \\
i\end{array}$} & \\
\hline & MORT & NEON & SUB $\mathrm{i}$ & MORT & NEON & SUB T & & & & \\
\hline VERTICE & 13 & 7 & 20 & 4 & 3 & 7 & 91 & 558 & 649 & 676 \\
\hline CARA & & & & & 1 & 1 & 1 & 1 & 2 & 3 \\
\hline FRENTE & & & & & & & 1 & & 1 & 9 \\
\hline PELVIS & 3 & 3 & 6 & 3 & 1 & 4 & 6 & 13 & 19 & 29 \\
\hline HOMBRO & 3 & 8 & 4 & 2 & & 2 & 3 & 8 & 19 & 17 \\
\hline TOTAL & 19 & 19 & 30 & 9 & 5 & 14 & 102 & 580 & 682 & 726 \\
\hline
\end{tabular}

A

\begin{tabular}{|c|c|c|c|c|c|c|c|}
\hline \multirow{2}{*}{ PRESENTACION } & \multicolumn{2}{|c|}{ PREMATUROS } & \multicolumn{4}{|c|}{ ATERMINO } & \multirow{2}{*}{ TOTAL } \\
\hline & VIVOS & MUERT & \multicolumn{2}{|c|}{ VIVOS } & \multicolumn{2}{|c|}{ MUERT } & \\
\hline VERTICE & 9139.2 & $2066 \%$ & 558 & 96.2 & 7 & 50 & 676 \\
\hline$C A R A$ & 10.98 & & 1 & 0.2 & 1 & 71 & 3 \\
\hline FRENTE & $9 \mid 0.98$ & & & & & & 1 \\
\hline PELVIS & 65.09 & $6 \longdiv { 2 0 }$ & 13 & 22 & 4 & 286 & 29 \\
\hline HOMBRO & $3 \mid 2.94$ & $4 \mid 133$ & 3 & 1.4 & 2 & 14.3 & 17 \\
\hline TOTAL & 102 & 30 & 580 & & 14 & & 726 \\
\hline
\end{tabular}

8

\begin{tabular}{|l|r|r|r|}
\hline PRESENT & No DECASOS & \multicolumn{2}{|c|}{ DEFUNCION } \\
\hline VERTICE & 676 & 27 & 4 \\
CARA & 3 & 1 & 33 \\
FRENTE & 1 & & \\
PELVIS & 29 & 10 & 34.5 \\
HOMBRO & 17 & 6 & 353 \\
\hline TOTAL & 726 & 44 & \\
\hline
\end{tabular}

En el cuadro No $10 \mathrm{~A}$ se muestra la incidencia de cada uno de los tipos de parto y su correspondiente porcentaje de mortalidad, de acuerdo a las diferentes intervenciones. Se observa que en forceps y vacuum la mortalidad fue de $0 \%$ en relación con las de cesárea $(17.5 \%)$ y versión $(25 \%)$. 
CUADRO Nㅇ 10

\begin{tabular}{|c|c|c|c|c|c|c|c|c|c|c|}
\hline \multirow{3}{*}{ TIPO DE PARTO } & \multicolumn{6}{|c|}{ DEFUNCIONES } & \multicolumn{3}{|c|}{ VIVOS } & \multirow{3}{*}{ TOTAL } \\
\hline & \multicolumn{3}{|c|}{ PREMATUROS } & \multicolumn{3}{|c|}{ A TERMINO } & \multirow{2}{*}{$\begin{array}{l} \\
\substack{a \\
\alpha \\
\alpha \\
a} \\
a\end{array}$} & \multirow{2}{*}{\begin{tabular}{|l|}
$\Sigma$ \\
$\alpha$ \\
$\alpha$ \\
\\
\\
\end{tabular}} & \multirow{2}{*}{\begin{tabular}{|l} 
\\
5 \\
0 \\
0 \\
0 \\
0
\end{tabular}} & \\
\hline & MORTIN & NEONAT & SUв тот & MORTIN & NNEONA & SUв тот & & & & \\
\hline ESPONTANEO & 14 & 09 & 23 & 6 & 3 & 9 & 90 & 504 & 594 & 626 \\
\hline CESAREA & 3 & 1 & 4 & 1 & 2 & 3 & 6 & 27 & 33 & 40 \\
\hline VACUUM & & & & & & & 1 & 17 & 18 & 18 \\
\hline VERSION & & 1 & 1 & & & & 1 & 2 & 3 & 4 \\
\hline FORCEPS & & & & & & & 4 & 30 & 34 & 34 \\
\hline EMBRIOTOMIA & 1 & & 1 & 2 & & 2 & & & & 3 \\
\hline CODUPLICATIO & 1 & & 1 & & & & & & & \\
\hline TOTAL & 19 & 11 & 30 & 9 & 5 & 14 & 102 & 580 & 682 & 726 \\
\hline
\end{tabular}

A

\begin{tabular}{|l|r|r|r|r|}
\hline TIPO DE PARTO & N:CASOS & \multicolumn{1}{|c|}{$\%$} & \multicolumn{2}{|c|}{ DEFUNCIONES } \\
\hline ESPONTANEO & 626 & 862 & 32 NN & $5.1 \%$ \\
CESAREA & 40 & 55 & 7 & 17.5 \\
VACUUM & 18 & 25 & & \\
VERSION & 4 & 0.6 & 1 & 25. \\
FORCEPS & 34 & 4.7 & & \\
EMBRIOTOMIA & 3 & 0.4 & & \\
CONDUPLICATIO & 1 & 0.1 & & \\
\hline TOTAL & 726 & & & \\
\hline
\end{tabular}

$B$

\begin{tabular}{|l|c|c|r|}
\hline CESAREA & N & \multicolumn{2}{|c|}{ DEFUNCIONES } \\
\hline FETO VIVO & 36 & 3 & $8.3 \%$ \\
\hline FETOMUERTO & 4 & \\
\hline TOTA L. & 40 & \\
\hline
\end{tabular}

C

\begin{tabular}{|c|c|c|}
\hline \begin{tabular}{|l} 
INTERV \\
FETO VIVO \\
\end{tabular} & NNO DEFUNCIONES & $\%$ \\
\hline 92 & 4 & 4.3 \\
\hline
\end{tabular}


En cuanto a las cesáreas, cuadro No $10 \mathrm{~B}$, se practicaron 40 discrimina. das en 36 en feto vivo, con una mortalidad de1 8,3\% ( 3 casos) y 4 en feto muerto antes de llegar al hospital.

Se practicaron un total de 99 intervenciones (13.6\%), cuadro $10 \mathrm{C}: 7$ en feto muerto ante-partum y $92 \mathrm{en}$ feto vivo, de los cuales murieron 4 , que representan un $4.3 \%$.

Estado al nacer: (Cuadro Nọ 11).

$C U A D R O$ №11

\begin{tabular}{|c|c|c|c|c|c|c|c|}
\hline \multirow{3}{*}{$\begin{array}{l}\text { ESTADO } \\
\text { AL NACER }\end{array}$} & \multicolumn{3}{|c|}{ PRE MATUROS } & \multicolumn{3}{|c|}{ A TERMINO } & \multirow{3}{*}{1014} \\
\hline & \multirow{2}{*}{\begin{tabular}{|c|}
$n$ \\
0 \\
$\vdots$ \\
\end{tabular}} & \multicolumn{2}{|c|}{ DFFUNT IONES } & \multicolumn{2}{|c|}{ DFFUACIONE: } & \multirow{2}{*}{$-1 \begin{array}{l}n \\
0 \\
\vdots \\
3\end{array}$} & \\
\hline & & MOR 1 & $N \in \cap n$ & $M \cap B S$ & NEON & & \\
\hline BUENO & 86 & & 0 & & & 566 & 652 \\
\hline & 12 & & 10 & & 2 & 11 & 35 \\
\hline & 4 & & 1 & & 3 & 3 & 11 \\
\hline MUERTE ANTEPARTO & & 16 & & 6 & & & 22 \\
\hline MUERIE INTRAPARTO & & 3 & & 3 & & & 6 \\
\hline TOTAL & 102 & 19 & 11 & 9 & 5 & 580 & 726 \\
\hline
\end{tabular}

A
\begin{tabular}{|l|c|c|}
\hline MORTINATOS & NOCASOS & $\%$ \\
\hline MUERTE ANTEPARTO & 22 & 786 \\
MUERTE INTRAPARTO & 6 & 214 \\
\hline TOTAL & 28 & \\
\hline
\end{tabular}

En el cuadro No 11 vemos la clasificación de los casos, en cuanto al estado al nacer.

En el cuadro No $11 \mathrm{~A}$, observamos la distribución de los mortinatos, de acuerdo al estado al llegar al hospital, y encontramos que de un total de 28 , el $78.6 \%$ (22 casos) murieron antes de llegar al hospital, y el $21.4 \%$ ( 6 casos) murieron durante el trabajo de parto o en el parto mismo. Relacionando este número con el total de la muestra, tenemos que en el $0.8 \%$ de nuestros mortinatos, su causa puede ser dependiente del manejo obstétrico.

\section{CAUSAS MEDICAS DE MORTALIDAD PERI-NATAL}

Al hacer el anáiisis de las causas médicas de mortalidad perinatal es necesario recordar algunos hechos importantes.

CUADR O N 112
\begin{tabular}{|l|r|r|r|}
\hline GRUPOS DE PESO & -2500 & +2501 & TOTAL \\
\hline MUERTE ANTEPARTO & $36 \%$ & $14 \%$ & $50 \%$ \\
\hline MUERTE INTRAPARTO & $7 \%$ & $7 \%$ & $14 \%$ \\
\hline MUERTE POST PARTO & $25 \%$ & $11 \%$ & $36 \%$ \\
\hline TOTAL & $68 \%$ & $32 \%$ & $100 \%$ \\
\hline
\end{tabular}

El cuadro No 12 muestra que 68\% de las defunciones ocurrieron en nacimientos prematuros.

Es además interesante que el $50 \%$ de las muertes peri-natales sucedieron ante-partum; 14\%, intra-partum; y $36 \%$ en el post-partum, hasta el $7^{\circ}$ día.

En el cuadro No 13 reunimos las causas médicas de muerte. Es necesario insistir en que para cada caso influyen varios factores. Nosotros hemos usado como criterio la estimación clínica, y anotamos una sola causa para cada caso. De esta estimación hemos se!eccionado la causa que consideramos decisiva o primitiva. Desde luego, hay un factor subjetivo en este modo de análisis, pero creemos que tiene la ventaja de poder indicar la base clínica que tiene el problema de la mortalidad peri-natal.

Clasificamos nuestros casos en 4 grandes grupos de anoxia: Anóxica, anémica, histotóxica y de causa desconocida. 


\begin{tabular}{|c|c|c|c|c|c|c|c|c|}
\hline \multirow{3}{*}{$\begin{array}{l}\text { CAUSAS MEDICAS } \\
\text { OE MUERTE } \\
\text { ANOX\|A }\end{array}$} & \multicolumn{6}{|c|}{ DEFUNCIONES } & \multirow{2}{*}{\multicolumn{2}{|c|}{ TOTAL }} \\
\hline & \multicolumn{3}{|c|}{ PREMATUROS } & \multicolumn{3}{|c|}{ A TERMINO } & & \\
\hline & MORT & NEON? & suB & MORTI & NEON? & SuB & No & $\%$ \\
\hline ANOXIA ANOXICA & & & & & & & & \\
\hline ATELECTASIA PULMONAR & & 6 & 6 & & & & 6 & 136 \\
\hline BRONCONEUMONIA Y NEUMONIA & & 3 & 3 & & 1 & 1 & 4 & 9 \\
\hline CIRCULARES Y PROLAPSO DEL CORDON & 3 & & 3 & 1 & 1 & 2 & 5 & 114 \\
\hline TOXEMIA & 1 & & 1 & & 1 & 1 & 2 & 45 \\
\hline TRAUMATISMO OBSTETRICO & & & & 2 & 1 & 3 & 3 & 68 \\
\hline SITUACION TRANSVERSA & & 1 & 1 & 2 & & 2 & 3 & 68 \\
\hline ABUSOS DE OCITOCICOS & 1 & & 1 & 1 & & 1 & 2 & 45 \\
\hline MALFORMACIONES & & & & & 1 & 1 & 1 & 23 \\
\hline POST MADUREZ & & & & 1 & & 1 & 1 & 23 \\
\hline SUB TOTAL & & & 15 & & & 12 & 27 & 61.2 \\
\hline ANOXIA ANEMICA & & & & & & & & \\
\hline ABRUPTIO PLACENTA & $?$ & & 7 & & & & 7 & 16 \\
\hline PLACENTA PREVIA & 2 & & 2 & & & & 2 & 45 \\
\hline RUPTURA UTERINA & 1 & & 1 & & & & 1 & 23 \\
\hline SUB TOTAL & & & 10 & & & & 90 & 22.8 \\
\hline ANOXIA HISTOTOXICA & & & & & & & & \\
\hline SEPTICEMIA & & 1 & 1 & & & & 1 & 23 \\
\hline LUES & 1 & & 1 & & & & 1 & 23 \\
\hline SUB TOTAL & & & 2 & & & & 2 & 4.6 \\
\hline ANOXIA DE CAUSA DESCONOCIDA & 3 & & 3 & 2 & & 2 & 5 & 11.4 \\
\hline SUB TOTAL & & & 3 & & & 2 & 5 & 11.4 \\
\hline TOTAL & 19 & 11 & 30 & 9 & 5 & 14 & 44 & \\
\hline
\end{tabular}

En el cuadro observamos que el mos incriminar a problemas de venti$61 \%$ (27 casos) corresponden a anoxia lación pulmonar (bronconeumonía, neuanóxica; $23 \%$ (10 casos), a anoxia monía y atelectasis) un total de 10 anémica; 5\% (2 casos), a histotóxica casos, correspondientes a $22,6 \%$ de y $11 \%$ ( 5 casos) en que la causa de los casos. anoxia no pudo ser establecida.

Analizando cada uno de los grupos vemos que por anoxia anóxica pode.

A causa netamente obstétrica (traumatismo y situación transversa) co- 
rresponden 6 casos, lo que significa un $13.6 \%$.

A interferencia de la circulación materno-fetal (circulares y prolapso del cordón), 5 casos, que significan el $11.4 \%$ de la mortalidad.

Es de anotar que la toxemia sigue siendo causa importante de defunciones con 2 casos, o sea el $4.5 \%$ de las muertes.

Hemos clasificado el abuso de ocitócicos dentro de este grupo, ya que pudimos comprobar en los casos correspondientes, anomalías de la contracción uterina, que significaron grave interferencia en la circulación placentaria. Estos casos fueron tratados por comadronas fuera del hospital, con grandes dosis de ocitócicos, a los cual achacamos la muerte intra-partum. Son 2 casos que representan $4.5 \%$ de las defunciones. Podemos ar̂irmar que las comadronas, en nuestro medio, mataron tantos niños como la toxemia.

Las malformaciones y la post-madurez son responsables, con un caso cada una, del $2.3 \%$ de las defunciones para cada una de estas circunstancias.

Anoxia anémica. Hemos clasificado en este grupo el abruptio placentae, la placenta previa y la ruptura uterina, ya que la pérdida de sangre materna directamente va a afectar el volumen sanguíneo del feto.

De abruptio placentae responsabilizamos 7 defunciones, o sea el $16 \%$ de las muertes.

La placenta previa produjo 2 defunciones, o sea el $4.5 \%$ de las muertes.
Tuvimos un caso de muerte por ruptura uterina, que representa el $2.3 \%$ de las defunciones.

Anoxia histotóxica. Clasificamos en este grupo un caso de septicemia y uno de lúes, que corresponde al $2.3 \%$ cada uno.

Anoxia de causa desconocida. En este grupo encontramos 5 casos correspondientes al $11.4 \%$ de las defunciones. Todas ellas sucedieron antes de la admisión de las madres en el hospital, y no nos fue posible encontrar una causa para explicarlas. Es posible que un estudio post-mortem de ellos nos hubiera dado información valiosa.

Nos ha parecido interesante presentar algunas asociaciones de factores que se añadieron a la causa principal de muerte.

En el grupo de anoxia anóxica encrintramos:

Atelectasia. Un caso con hemorr:tgia cerebral y prematurez, que vivió un día.

Un caso con hemorragia sub-aracnoidea y prematurez, que vivió 3 días.

Un caso con placenta previa y prematurez, que vivió 6 días.

Un caso con presentación de pelvis y operación cesárea, que vivió un día.

Dos casos de prematurez, que vivieron un día y 7 días cada uno.

Bronconeumonía y neumonía. Dos casos con ruptura prematura de membrana e infección amniótica.

Dos casos con aspiración de líquido amniótico. 
Trauma obstétrico. Tres casos de retención de cabeza última, en partos en presentación de pelvis.

Situación transversa. Tres casos de presentación de hombro, que se manejaron así: uno por versión y extracción en un segundo gemelo prematuro y los otros dos por embriotomía.

Circulares y prolapso del cordón. Cinco casos que sucedieron con ruptura prematura de membranas. Este accidente se produjo antes del ingreso de las pacientes al hospital, en 4 casos; 2 de ellos se asociaron con presentación de pelvis en prematuro. Un caso con situación transversa en prematuro y otro sin ninguna patología asociada. Un caso en que hubo circulares apretadas al cuello.

Toxemia Uno de los casos fue de eclampsia antes de la hospitalización de la paciente y presentación de pelvis.

El otro de pre-eclampsia severa en un prematuro.

Abuso de oxitócicos. Es necesario hacer notar que en ambos casos hubo desprendimiento parcial de la placenta, achacable al mal manejo de los oxitócicos.

Malformaciones. El caso de malformaciones que encontramos fue una hidrocefalia, que se asoció con presentación de cara.

Los casos de anoxia anémica se asociaron así:

Abruptio placentae, un caso con pre-eclampsia severa.
Un caso con pre-eclampsia severa y situación transversa.

Es de anotar que los 7 casos de abruptio analizados fueron mortinatos prematuros, 5 de ellos asociados a gran multiparidad.

Placenta previa. Un caso de placenta previa, $100 \%$ en presentación de pelvis y prematurez.

El otro de placenta previa, $20 \%$, fue prematuro.

Ruptura uterina. Se debió a la ruptura espontánea de cicatriz de 3 cesáreas anteriores, complicada con situación transversa, que sucedió antes de su ingreso al hospital, en trabajo de parto prematuro.

En el grupo de anoxia histotóxica anotamos:

Septicemia, un caso asociado a ruptura prematura de membranas e infección amniótica.

Lúes. Un caso, sin control prenatal, mortinato, con lesiones luéticas múltiples.

Es interesante presentar nuestras cifras con respecto a ruptura prematura de membranas, ya que esta condición, aunque no es directamente responsable de la muerte peri-natal, se asocia con condiciones que la hacen merecer un comentario especial.

De un total de 42 casos de ruptura prematura de membranas de nuestra muestra, el $47.6 \%$ hizo infección amniótica, con una mortalidad peri-natal de $6.4 \%$, representada por una septicemia y dos bronconeumonías; $9.5 \%$ se complicaron con prolapso de cordón. 
Dada la importancia que tiene la toxemia entre nosotros, destacamos también los siguientes hechos: en la muestra de 726 nacimientos tuvimos $8 \%$ de toxemias, que nos produjeron una mortalidad peri-natal de $3.5 \%$.

Destacamos también que en la muestra hubo un $1.5 \%$ abruptio placentae (11 casos), con un $64 \%$ de mortalidad.

\section{CUADRO Nㅇ 14}

INDICE MORTALIDAD PERINATAL

$$
\begin{aligned}
& \frac{\text { MUERTES PERINATALES }}{\text { NACIDOS VIVOS }} \times 1000=63 \% \\
& \text { INDICE PREMATUREZ } \\
& \frac{\text { PREMATUROS }}{\text { NACIDOS VIVOS }} \times 1000=189 \% \\
& \text { INDICE MORTINATALIDAD } \\
& \frac{\text { NACIDOS MUERIOS }}{\text { NACIDOS VIVOS }}=4000=40 \% \\
& \text { INDICE DE MORTALIDAD NEONATAL } \\
& \text { HASTA EL 7DIA } \\
& \frac{\text { MUERTES HASTA 7\%DIAS }}{\text { NACIDOS VIVOS }}
\end{aligned}
$$

\section{COMENTARIOS}

Los datos presentados nos permiten obtener los coeficientes que presentamos en el cuadro $N^{\circ} 14$.

Un análisis de ellos nos permite hacer notar que el coeficiente de mortalidad perinatal, que es del $63 \times 1.000$, es alto. Es importante destacar que la mortalidad es del 40 x 1.000 , y la mortalidad neo-natal, hasta el $7^{0}{ }^{0}$ día, es de $23 \times 1.000$.
Un factor que a nuestro modo de ver influye sobre la mortalidad perinatal es la gran incidencia de prematurez, que alcanza 189\%. Las cifras de prematurez, así como la alta incidencia de defunciones tanto de mortinatos como neonatos en este mismo grupo, nos inclinan, desde un punto de vista práctico, a estudiar la forma de poder disminuír la mortalidad peri-natal influyendo sobre las causas demográficas y medidas que inciden sobre el problema.

En el análisis de los factores demográficos presentados, se observó claramente la influencia de la edad, paridad y gravidez sobre la incidencia de mortalidad en nuestra serie. Si a estos datos agregamos el factor control prenatal, creemos que la falta de vigilancia adecuada durante el transcurso del embarazo es un factor de gran significación.

Lograr que las mujeres asistan a consulta prenatal es una empresa difícil en nuestro medio. Nos parece que hay varias razones para que no lo hagan, siendo la principal la falta de educación. Las clases sociales altas en general, conocen el problema y buscan la asistencia médica necesaria. No así las clases sociales bajas, porque no han sido enseñadas al respecto.

Se hace necesario organizar facilidades asistenciales y económicas para que la embarazada tenga vigilancia médica durante la gravidez. Las dificultades nacen de consultorios prenatales insuficientes, que significan para la mujer larga espera y pérdida de tiempo, que la impiden atender sus obligaciones en el hogar o en el trabajo. 
Muchas de las condiciones médicas y obstétricas que complican el embarazo y el parto pueden ser descubiertas y tratadas mediante el control prenatal, de modo que haga favorable su evolución, y en esa forma disminuír la rata de prematurez $\mathrm{y}$, por ende, la de mortalidad peri-natal.

En nuestras series creemos que un $50 \%$ de las defunciones hubieran podido ser prevenidas mediante el diagnóstico adecuado durante el embarazo, y en un alto porcentaje prevenido el parto prematuro en nuestras pacientes.

En cuanto a causas médicas de mortalidad peri-natal podemos concluír:

Las condiciones maternas contribuyeron en un $30 \%$ a la mortalidad, y las complicaciones del trabajo y del parto, en un $34 \%$. El resto se distribuyen en un $23 \%$ para entidades pulmonares del recién nacido, un $2 \%$ para malformaciones y un $11 \%$ para causas desconocidas.

\section{RECOMENDACIONES}

Con la intención de buscar solución a los factores y causas que inciden sobre la mortalidad perinatal, creemos necesario actuar en diferentes campos.

19 Mejorando el control prenatal, facilitando la consulta y logrando la hospitalización oportuna de los casos que constituyen mayor riesgo o complicados.

$2^{\circ}$ Haciendo extremos los cuidados técnicos de la atención del parto y el buen tratamiento de las complicaciones del mismo.
30 Mejorando la prevención y el tratamiento de complicaciones en el recién nacido a término y prematuro.

49 Con miras a un mejor estudio de las causas de mortalidad peri-natal, recomendar unificar en el país los certificados de defunción y las historias clínicas en los servicios asistenciales. En esa forma se podrá algún día ha. cer estudios completos a nivel nacional.

$5^{\circ}$ Con miras al mejor conocimiento de las causas de mortalidad, insistir en la coordinación de los servicios obstétricos y de recién nacido con los de patología, con el objeto de obtener exámenes post-mortem y examen histopatológico de la placenta.

\section{COLABORARON \\ A ESTE TRABAJO:}

\section{Doctor Bernardo Aguilera Camacho.} Profesor Jefe de la Sección de Estadística del Departamento de $\mathrm{Me}$ dicina Preventiva y Salud Pública. Universidad del Valle. Facultad de Medicina.

Doctor Carlos Goubert. Residente del Departamento de Obstetricia y Ginecología. Universidad del Valle. Facultad de Medicina.

Personal de tabulación del Departamento de Medicina Preventiva y Salud Pública. Universidad del Valle. Facultad de Medicina.

A quienes los autores manifiestan sus agradecimientos. 


\section{BIBLIOGRAFIA}

1. AHVEnAINEN E. K. A.: Study of the causes of perinatal death Acta Obst. et Gynec. Scandinav. 39: 438, 1960.

2. BAIRD D.: The influence of social and economic factors on stillbirths and neo-natal deaths. J. Obst. \& Gynec. Brit. Emp. 52: 217, 1945.

3. BUNDESEN H. N., POTTER E. L. FISHBEIN, W. I. BAUER F. C. and PLOTZKE G. V. Progress in the prevention of needless neo-natal deaths. Chicago. 1951.

4. CORREA PELAYO, CASTRO SALVADOR: Hallazgos patológicos en muertes peri-natales. Antioquia Médica. 9: 59. 1959.

5. DAVIS M. E. and POTTER E. L.: Congenital malformations and obstetrics Pediatrics. 19: 719. 1957.

6. D'ESOPO D. A. and MARCHETT A. A.: Causes of fetal and neo-natal mortality. Am. J. Obst. \& Gynec. 49: 1. 1952.

7. EASTMAN N. J.: Williams Obstetrics. New York. Appleton Century-Crofts. 1956.

8. FRAZIER T. M., NESBITT R. E. L., Jr., and PENTECOST M. P., Jr.: Accuracy of the reported causes of fetal and neo-natal deaths. Pub. Health Rep. 72: 933. 1957.

9. HARDY J. B.: Peri-natal mortality. Clin. Obst. \& Gynec. 5: 141. 1962.

10. HEADY J. A., STEVENS C. F., DALY C. and MORRIS J. N.: Social and biologic factors in infant mortality. IV. The independent effects of social class, religion, the mother's age and her parity. Lancet 268: 499. 1955.

11. HELLMAN L. M.: The prevention of premature birth, in prematurity, congenital malformation, and birth injury. New York. 1953. Association for the Aid of Crippled Children.

12. HOLZAEPFEL J., RANNEY B., NICOLAY K.: An outline for the organization of peri-natal mortality studies. Am. J. Obst. \& Gynec. 81: 906. 1961.

13. HUNT E. P. and CHENOWETH A. D.: Recent trends in infant mortality in the United States. Am. J. Pub. Health. 51: 190. 1961.

14. KOHL S. G.: Peri-natal mortality in New York City. Harvard University Press. Cambridge, Mass. 1955.

15. NESBITT R. E. L., Jr.: Peri-natal lost in modern obstetrics. Davis. Philadelphia. 1957.

16. POTTER E. L. and ADAIR F. L.: Fetal and neo-natal death. Edit. Chicago. 1949.

17. ROCHESTER A.: Infant mortality. Children's Bureau Pub. 119. 1923.

18. SHWARTZ S.: Pre-natal care, prematurity and neo-natal mortality. Am. J. Obst. \& Gynec. 83: 591. 1962.

19. STEWART A.: A note on the obstetric effect of work during pregnancy Brit. J. Prev. \& Social Med. 9: 159. 1955.

20. TAYLOR E. S. and WALKER L. C.: Premature infant. deaths. A ten year study of causes and prevention. Obst. \& Gynec. 13: 555. 1959.

21. TEIT KAERN: Peri-natal mortality. Acta Obst. et Gynec. Scandinav. 39: 392. 1960. 